\title{
La comunicación sobre la salud en medios digitales cubanos
}

\author{
Health communication in the Cuban digital media
}

\begin{abstract}
Alina Pérez Martínez
Doctora en Medicina. Especialista de I Grado en Medicina General Integral. Máster en Ciencias de la Comunicación. Centro Nacional de Información de Ciencias Médicas. La Habana, Cuba.
\end{abstract}

\section{RESUMEN}

I ntroducción La creación de una revista electrónica de salud dirigida al público no especializado requiere de conocer los antecedentes del tratamiento de la temática en las publicaciones digitales nacionales.

Objetivo Caracterizar la divulgación sobre salud en documentos seleccionados de siete web periodísticas cubanas: Granma, Juventud Rebelde, Trabajadores, Bohemia, Mujeres, Alma Mater y Somos J óvenes, publicados en abril de 2009.

Métodos Estudio descriptivo de casos múltiples con técnicas de investigación documental y de análisis de contenido bajo una perspectiva cualitativa.

Resultados El enfoque de divulgación no fue sustancial, los contenidos no respaldaron las estrategias para la promoción de la salud y en su mayoría no guardaron relación con las prioridades sanitarias del Ministerio de Salud Pública cubano. Dentro de la minoría que abordó contenidos priorizados, no se encontraron temas relacionados con el agua, saneamiento ambiental y control de vectores; el consumo de drogas, trastornos mentales, enfermedades bucodentales, discapacidad, salud escolar y del trabajador, atención al adulto mayor, entre otros. Solo el 5,8 \% de estos artículos se refirió a políticas de salud, pero abordadas de forma implícita y no explicada desde una perspectiva científica. Predominó el enfoque curativo.

Conclusiones La temática sobre salud en los documentos estudiados no se enfoca con profundidad hacia la divulgación de salud, no respalda a las estrategias para la promoción de la salud ni las prioridades del MINSAP.

Palabras clave: Promoción de salud, salud pública, comunicación social, medios de comunicación, periodismo especializado en salud, divulgación científica, evaluación de 
la comunicación, informetría, medios digitales, publicaciones electrónicas, publicaciones seriadas, web periodísticas.

\begin{abstract}
Introduction The creation of an electronic health journal aimed at the general nonspecialized audience requires knowing the previous history of this topic in the national electronic publications.

Objective to characterize the dissemination of health information in selected documentes from seven Cuban press websites: Gramma, Juventud Rebelde, Trabajadores, Bohemia, Mujeres, Alma Mater and Somos Jóvenes, all published in April 2009.

Methods Descriptive study of multiple cases using documentary research and content analysis techniques under a qualitative perspective.

Results The approach to health dissemination was not significant, the contents did not support health promotion strategies and most of them did not relate with the health priorities of the Ministry of Public Health in Cuba. Among those that dealt with priority contents, there were no topics about water, environmental protection and vector control, drug addiction, mental disorders, oral and dental diseases, disability, health at school, occupational health, care of the older adult and others. J ust $5.8 \%$ of these articles reflected health policies but they were addressed implicitly rather than explicitly from a scientific perspective. The curative approach was predominant. Conclusions Health topics in the studied documentes neither focused on health dissemination nor backed the health promotion strategies and priorities of the Ministry of Public Health.
\end{abstract}

Key words: Health promotion, public health, social communication, communication media, health-specialized journalism, scientific diffusion, communication assessment, informetrics, digital media, electronic publications, serial publications, press Websites.

\title{
I NTRODUCCI ÓN
}

Cuando se planteó convertir el sitio web "Salud Vida" de la Red de Salud de Cuba, Infomed, en una publicación electrónica seriada con un enfoque de prevención y promoción de salud, dirigida a un público no especializado, apremiaba la necesidad de profundizar en la temática de salud en los medios digitales cubanos, como referentes obligados para valorar su pertinencia, y redimensionarla a partir de una caracterización de la divulgación de salud en estas publicaciones.

Inicialmente se buscaron aquellas publicaciones digitales que pudieran compartir similar intención editorial en relación con la salud. Al consultar el Catálogo Nacional de Publicaciones Seriadas Cubanas, se encontró que existen en Cuba 77 publicaciones electrónicas de temas de medicina y salud pública, pero ninguna dirigida a un público no especializado. Se revisó entonces, el resto de las publicaciones electrónicas seriadas registradas, que suman en total 341 y no existe ningún sitio web registrado dedicado exclusivamente a la divulgación de salud en general para el público no 
especializado, pero sí en las más importantes publicaciones digitales cubanas, aparece el tema en secciones o espacios eventuales. ${ }^{1}$

Los objetivos propuestos para esta investigación fueron: determinar los contenidos temáticos de divulgación sobre salud en medios digitales cubanos seleccionados, y su relación con las áreas prioritarias del Ministerio de Salud Pública (MINSAP) y definir los tipos de contenidos de divulgación de salud en las web seleccionadas y su orientación según el enfoque fuera curativo, preventivo o de promoción de salud.

\section{MÉTODOS}

Se realizó un estudio de casos múltiple de carácter empírico-descriptivo, bajo una perspectiva cualitativa. Las técnicas de investigación utilizadas fueron la investigación documental o bibliográfica, el análisis de contenido y el análisis de una web periodística.

Las categorías de análisis fueron: el enfoque del mensaje hacia la divulgación científica, los tipos de contenidos sobre salud y la presencia de las proyecciones sanitarias priorizadas para el 2015. El enfoque predominante de los contenidos: curativo, preventivo o de promoción de salud. Las estrategias de la promoción de la salud: políticas públicas favorables, fortalecimiento de la acción comunitaria, desarrollo de aptitudes personales, creación de entornos propicios y reorientación de los servicios. ${ }^{2,3}$

Se realizó una selección intencional del universo de publicaciones seriadas nacionales, disponibles en la web, y dirigidas al público no especializado. Fueron seleccionadas Granma, Juventud Rebelde, Trabajadores, Alma Mater, Bohemia, Mujeres y Somos Jóvenes. Las unidades de análisis la constituyeron 45 trabajos relacionados con la salud humana en el mes de abril de 2009 (anexos 1 y 2)

\section{RESULTADOS}

En relación con los contenidos de salud de las web seleccionadas se encontraron 45 trabajos que abordan directa o indirectamente temas de salud. Al analizar los contenidos de estos trabajos, solo 34 de ellos $(75,5 \%)$, lo hacen desde el enfoque de la divulgación de salud, pues el resto no explican o difunden información relativa al conocimiento, la cultura o el pensamiento cientificotécnico en el campo de la salud, sino que se orientan hacia la información de tipo general.

Los términos, "Cuba" y "cubanos" en los titulares de los trabajos sobre salud, aparecieron en 11 documentos (24,4 \%), 8 (50 \%) de ellos en Granma. También en los titulares se utilizaron términos que refuerzan en sentido positivo los logros de la salud cubana, tales como: generalizará, buenos resultados, eficacia, desarrollan, refuerzan, últimos avances, exitosa, garantizada, aportes; no existen, términos o contenidos con una visión negativa o de cuestionamiento en relación con la salud o la salud pública en Cuba.

Entre los trabajos que tienen un enfoque de divulgación de salud, no todos lo hacen en igual magnitud, por lo que fue importante considerar 13 trabajos $(28,8 \%)$ que solo contienen escasos elementos de divulgación y otros 21 (46,6\%), que son predominantemente de divulgación; todos los casos estudiados de la revista Mujeres 
pertenecen a este último grupo, seguidos de Juventud Rebelde con la mayoría de sus trabajos sobre salud, con un enfoque mayormente de divulgación.

En relación con los contenidos temáticos de divulgación sobre salud, los temas más tratados fueron las enfermedades crónicas no trasmisibles como la enfermedad isquémica del corazón y los tumores malignos; en segundo lugar, la hipertensión arterial, las enfermedades cerebrovasculares y el asma bronquial, junto a enfermedades trasmisibles como el VIH/sida, y enfermedades eliminadas o controladas por vacunas. Aparecieron también contenidos relativos a la atención a la infancia y a la prevención de la mortalidad materna, todos estos relacionados directamente con las prioridades del MINSAP.

Por otra parte, se encontraron temas muy diversos, que en el 55, $8 \%$ de los casos, no se relacionaban con las áreas prioritarias del MI NSAP. Así se hace referencia al programa cubano de trasplantes, al veneno del escorpión azul, a infecciones de transmisión sexual diferentes del $\mathrm{VIH} /$ sida, a la planificación familiar, a las bondades del yogurt o a la diversidad sexual.

En relación con los temas prioritarios del MINSAP, no se encontraron contenidos temáticos referidos a los factores relacionados con el ambiente, como el agua, el saneamiento ambiental y el control de vectores (mosquito); factores relacionados con el comportamiento tales como el tabaquismo, el alcoholismo, las drogas, la dieta y el sedentarismo, y enfermedades bucodentales. Están totalmente ausentes temas como los trastornos mentales, las lesiones autoinfligidas, la discapacidad motora, visual y auditiva, la salud escolar y de los trabajadores y de forma especial llama la atención la ausencia de contenidos relativos al adulto mayor.

En cuanto a los tipos de contenidos, la mayor cantidad de artículos, $26,4 \%$, están dirigidos a la educación para la salud y difunden información para facilitar el cambio de aptitudes, habilidades y prácticas en los individuos y comunidades, fundamentalmente en relación con la prevención de infecciones de transmisión sexual y hábitos nutricionales.

Los contenidos relacionados con la asistencia sanitaria y servicios de salud correspondieron al 20,5 \% y se refieren en su mayoría a servicios de atención terciaria y secundaria de salud; solo los relacionados con la vacunación antipolio y la planificación familiar, pertenecen al contexto de la atención primaria de salud.

El $14,7 \%$ de los casos correspondieron a temas de ciencias e investigaciones biomédicas, e igual porcentaje se refieren a fármacos, productos y tecnologías de la salud, tales como vacunas y productos naturales, en ambos casos muy vinculados a la investigación científica.

En estos trabajos se hace evidente la existencia de políticas públicas que favorecen la investigación biomédica y el interés por la generalización de sus resultados y el desarrollo de los servicios sanitarios y aún cuando solo el 5,8 \% se refiere a políticas de salud en Cuba, estas no son abordadas de forma explícita. Las políticas de salud están por tanto implícitas, pero no son suficientemente tratadas como tema central de estos trabajos, ni son explicadas desde una perspectiva científica. Teniendo en cuenta las implicaciones sociales de estas políticas públicas, no son utilizadas las ciencias sociales para explicarlas.

Se encontró un ejemplo de trabajo que se refiere a la formación de personal en ciencias de la salud y otro a la economía de la salud, que tratan estos temas de manera muy superficial. 
Es de destacar que si bien solo dos de los documentos estudiados se refieren directamente a eventos científicos de salud, el $29,4 \%$ de los trabajos hacen alusión a estos eventos de forma destacada.

No se encontraron trabajos de divulgación relacionados con la cooperación de salud de Cuba en el exterior.

\section{La visión hacia los nuevos paradigmas de la salud}

En relación con el enfoque de los contenidos según el paradigma de la salud, el $47 \%$ de los temas analizados tienen un marcado enfoque curativo, mientras el $35,2 \%$ se orienta a contenidos desde una óptica de prevención y solo el $17,6 \%$ lo hace mirando hacia la salud, desde la perspectiva de la promoción de la salud.

En cuanto al punto de vista de los contenidos según las estrategias para la promoción de la salud, el $38,2 \%$, están dirigidos a brindar información para el desarrollo de aptitudes personales.

Aún cuando no se encontraron alusiones explícitas a programas políticos y legislaciones en pro de la salud, que permitan una mayor equidad de salud, la existencia de políticas públicas favorables a la salud es indiscutible por el hecho de que se desarrollan investigaciones y producciones cubanas de todo tipo: productos para el cáncer, productos naturales, biomateriales para cirugía, software para señales electrocardiográficas, reactivos para el sida, las cuales se realizan en lugares diferentes, Santa Clara, Santiago de Cuba, La Habana, lo que denota una voluntad política nacional de desarrollo científico en la biomedicina. Trabajos como Santiago de Cuba: Sustituirán importación de hemoclasificadores y reactivos para el SIDA, demuestran indirectamente que estos reactivos, altamente costosos, se adquieren en el exterior para los pacientes que lo demandan. No siempre es explícito en los trabajos que estas investigaciones y producciones son todas estatales y de distribución gratuita, y sí es innegable que sus resultados serán generalizados a todo el país. También la generalización de servicios, como los de planificación familiar y vacunación, y otros de alta tecnología como la colocación de prótesis arteriales en el corazón, denotan un alto grado de equidad en el acceso y oportunidades en cuanto a la atención de salud.

En relación con cambios organizativos en respuesta a políticas públicas, es de señalar el trabajo Garantizada la atención al paciente grave, el único donde se hace explícita una política, en este caso para garantizar estos servicios con calidad en todo el país, llama la atención que se refiere a un servicio de tipo curativo.

No se encontraron artículos en que se abordara la participación efectiva y concreta de la comunidad en la fijación de prioridades, la toma de decisiones y la elaboración y puesta en marcha de estrategias de planificación para alcanzar un mejor nivel de salud, ni siquiera de forma implícita.

En general, no se hallaron contenidos que favorecieran el empoderamiento de los individuos, con la puesta en práctica de procesos participativos. Una excepción en este sentido lo constituyó el trabajo Sabia la naturaleza, que nos hizo diferentes, que informa sobre la conferencia de prensa ofrecida por Mariela Castro Espín, directora del Centro Nacional de Educación Sexual (CENESEX), para presentar las actividades de la campaña anual contra la homofobia, en la que afirma, "Aunque persisten abstenciones y actos en contra de las jornadas por la libre orientación sexual, a cada edición de estas se suman más asociaciones e instituciones políticas y culturales." 
En cuanto a la creación de otros entornos saludables, solo algunos ejemplos puntuales abordan indirectamente esta estrategia, sobre todo en relación con entornos sociales, como el citado caso de la diversidad social y el apoyo a mujeres con cáncer de mama, en el trabajo Por la vida.

En relación con la estrategia de la promoción de la salud llamada a reorientar los servicios de salud, el $32,3 \%$ de los casos estudiados son referidos a servicios médicos, pero se trata de servicios tradicionales dirigidos a la curación con introducción de nuevos productos y tecnologías o en un caso con un sentido de prevención de riesgos.

\section{DISCUSION}

\section{El enfoque de divulgación}

El concepto de divulgación científica se ha definido como "toda actividad de explicación y difusión de los conocimientos, la cultura y el pensamiento científico y técnico", siempre que esa difusión sea hecha fuera de la enseñanza oficial o equivalentes y que no tenga el objetivo de formar o capacitar a especialistas. ${ }^{4,5}$

La comunicación pública de la ciencia, a través de los medios de comunicación de masas, tiene la función de trasmitir y hacer comprensible el contenido de la ciencia, estimular el interés público, la sensibilidad y la responsabilidad moral, además de significar el sentido de los descubrimientos y de sus aplicaciones, especialmente los que tienen gran incidencia en la vida cotidiana. También pretende incidir en que las decisiones políticas se tomen teniendo en cuenta los avances científicos y tecnológicos, con la mirada puesta en el ser humano, al servicio de su calidad de vida y su enriquecimiento cultural. ${ }^{6-8}$

El hecho de que varios trabajos sobre salud no aborden contenidos de divulgación científica o que son escasos, denota que el enfoque prevalente del mensaje está dirigido a la información general o a la propaganda, lo cual coincide con lo encontrado por Diana Shlashter en sus estudios sobre la representación de la ciencia en medios de comunicación.

Los términos utilizados en los titulares denotan la intención de exaltar la imagen de Cuba a través de sus logros y resultados positivos en el campo de la salud, lo cual pudiera corresponderse con una intención editorial de estas publicaciones pero que se distancia de las funciones de la comunicación de la ciencia en los medios, antes descritas.

Por su parte, se afirma que "el proceso de intercambio de información capaz de generar retroalimentación modificando a los sujetos participantes, constituye el fenómeno conocido como comunicación" y reconoce que la comunicación masiva "despierta interés, crea opinión e imagen y predispone al público hacia conductas saludables". 9,10

En febrero de 2006, se publica el documento Proyecciones de la Salud Pública en Cuba para el 2015, en el que se proyecta: "una estrategia a largo plazo que considera, de manera integral, todos los componentes y determinantes del Estado de Salud de la Población, con un enfoque de riesgo". Este documento, establece ocho 
"áreas prioritarias", dirigidas en general, a modificar la morbilidad y mortalidad por enfermedades frecuentes. ${ }^{11}$

El hecho de que en los artículos analizados se traten temas que no se consideran entre los propósitos específicos de las áreas prioritarias del MINSAP, al menos no directamente, no significa que no sean temas necesarios y de interés del público. Recordemos que en este estudio se examina el contenido temático de los mensajes y su relación con las prioridades del MINSAP explícitas en su documento metodológico rector, pero no se incluyen otros documentos como los Programas Nacionales de Salud, ni se estudian estos contenidos desde las necesidades del público. No obstante, lo más significativo no es que la mayoría de los documentos no respondan a los temas de mayor prioridad del MINSAP, sino que hay gran cantidad de temas prioritarios que no están tratados.

La falta de correspondencia entre lo que se publica y las prioridades del MINSAP, pudiera deberse a una falla en la implementación de políticas de comunicación en el sector de la salud y de trabajo colegiado entre las entidades del MINSAP y los medios de comunicación que profesionalice el acceso a fuentes especializadas y la consecución de agendas en el campo de la comunicación social de la ciencia, lo cual trasciende los propósitos de este trabajo, para adentrarse en el ámbito de las ideologías profesionales y sus rutinas productivas.

Por otra parte, si se observan los contenidos temáticos abordados, se acercan más a la situación de salud y a las necesidades informativas de países desarrollados que a las de Cuba, se mezclan elementos comparables con estos países pero que coexisten con los determinantes sociales propios de un sistema social en vías de desarrollo, lo que pudiera explicarse a partir de una cierta idealización de la salud, que se manifiesta en los medios más como el tipo de situación de salud concebida como "buena" que como un reflejo objetivo de la realidad cubana.

En este mismo sentido cabría notar la "nobleza" de los temas abordados y mejor su aceptación por los lectores: las enfermedades crónicas trasmisibles o la salud materno infantil, por ejemplo, frente a los temas prioritarios ausentes. Estos últimos engloban a problemas de salud más "conflictivos" y menos agradables para el público, en los que se implicarían de forma indiscutible, las responsabilidades sociales e institucionales y no ya las de los individuos en el control de la salud.

Resultó inexplicable la ausencia de temas relacionados con la salud del adulto mayor, a pesar del proceso de envejecimiento poblacional y sus trascendencia de gran interés social y de la prioridad del tema por el MINSAP, lo que también pudiera denotar una contradicción entre las necesidades reales de información pública y su visibilidad en el discurso mediático.

La mención destacada a eventos científicos, evoca a un fenómeno señalado por otros autores como el "eventismo" en la divulgación de la ciencia y que pudiera responder, de una parte a la gran cantidad de eventos médicos que se realizan y por otra, a que esta sea una vía importante que utilizan los periodistas para acceder a la información.

El hecho de que la alusión a la formación de personal en ciencias de la salud y a la economía de la salud sea tan superficial, denota la falta de visión, en el momento de este estudio, de las profundas implicaciones que tanto la formación de recursos humanos como la economía de la salud tienen para el desarrollo y sostenibilidad de la salud pública cubana, en la situación socioeconómica interna del país y en la coyuntura internacional actual. 
La temática de la colaboración internacional de salud no está presente en los trabajos de divulgación científica, lo que indica que el tratamiento de estos temas se hace desde la información general, no especializada, pero no se realiza un análisis que afirme su trascendencia social o en la salud individual, desde una perspectiva científica, como pudiera hacerse desde el enfoque de la salud pública. Lo anterior es un vacío extraordinario si se considera que durante los últimos 10 años de forma estable, más de 30000 especialistas de la salud cubanos han prestados servicios en más de 50 países, con la consecuente trascendencia social y para la salud pública cubana e internacional que ello implica.

\section{La promoción de salud}

Durante siglos, la concepción de la salud era solo la ausencia de enfermedades y de invalidez, y el enfermo tenía un papel pasivo en la lucha contra su enfermedad. ${ }^{12,13}$

El llamado paradigma biomédico que comparte estos postulados, tiene una concepción biologicista, acultural, individualista y ahistórica de la salud-enfermedad, con una orientación predominantemente curativa. ${ }^{14}$

A mediados del siglo XX se comenzaron a plantear alternativas a esta perspectiva dada la reconsideración de otros factores implicados en la salud humana. Esto propicia la aparición de una nueva concepción de la enfermedad como fenómeno multicausal en el que los factores biológicos, conductuales y ambientales (físicos y sociales) cobran gran importancia. ${ }^{15-17}$

El análisis histórico de la salud y de su importancia para el bienestar humano realizado por Henry E. Sigerist, el gran historiador médico, citado por Toledo Curbelo, lo llevan a considerar la salud en un sentido social, sobre lo que afirmó:

Una persona sana es un ser humano con un buen equilibrio corporal y mental y bien adaptado a su medio físico y social. Ejerce pleno control de sus facultades físicas y mentales, puede adaptarse a los cambios ambientales siempre y cuando no sobrepasen los límites normales, y contribuye al bienestar de la sociedad en la medida de sus posibilidades. Por lo tanto, la salud no es sencillamente la ausencia de enfermedad; es algo positivo, una actitud alegre hacia la vida y la aceptación entusiasta de las responsabilidades que la vida impone a la persona. ${ }^{18}$

De este cambio en la concepción de la salud y enfermedad, da testimonio la definición del concepto de salud que la OMS acuñó en su Carta Constitucional de 1948. Según esta: La salud es el estado de completo bienestar físico, mental y social y no la mera ausencia de enfermedad. ${ }^{19}$

La nueva teoría de la promoción de la salud se inicia tras el cambio trascendental en el concepto de salud de la OMS de 1948. El paradigma biomédico había entrado en crisis y al decir de Helena Restrepo, "se ha generalizado un nuevo paradigma que considera a la salud como un concepto holístico positivo que integra todas las características del bienestar humano". ${ }^{20-22}$

La Carta de Ottawa, propone por primera vez un concepto amplio de gran consenso:

La promoción de la salud consiste en proporcionar a los pueblos los medios necesarios para mejorar su salud y ejercer un mayor control sobre la misma. Para alcanzar un estado adecuado de bienestar físico, mental y social un individuo o grupo debe ser capaz de identificar y realizar sus aspiraciones, de satisfacer sus necesidades y de cambiar o adaptarse al medio ambiente. La salud se percibe pues, no como el objetivo, sino como la fuente de riqueza de la vida cotidiana. Se trata por tanto de un 


\section{Revista Cubana de Salud Pública. 2011;37(3):288-305}

concepto positivo que acentúa los recursos sociales y personales así como las aptitudes físicas. Por consiguiente, dado que el concepto de salud como bienestar trasciende la idea de formas de vida sanas, la promoción de la salud no concierne exclusivamente al sector sanitario. ${ }^{23}$

La carta de Ottawa afirma que la promoción de la salud implica: la elaboración de políticas públicas saludables, el fortalecimiento de la acción comunitaria, el desarrollo de aptitudes personales, la creación de ambientes favorables a la salud, y la reorientación de los servicios sanitarios. ${ }^{24}$

En el caso de Cuba, según Toledo y otros:

[...] a pesar de que existen datos confiables de la situación de salud cubana desde la época colonial, la relación de esta situación con acciones promocionales no ha sido estudiada con frecuencia. Cuando se analiza la información disponible, es reiterativo el análisis del desarrollo del Sistema Nacional de Salud basado en el recuentro histórico de sus diferentes etapas y los consiguientes logros, pero escasean por no decir que no existen, los análisis originados en un enfoque más abarcador sobre las diferentes causas que han tenido esos efectos y en general se destaca poco la influencia de las acciones promocionales de responsabilidad estatal que tanto contribuyeron a proporcionar medios para la salud de los cubanos [... $]^{18,25}$

En el Sistema Nacional de Salud se crean diferentes estrategias de desarrollo como por ejemplo, la instauración en enero de 1984 del "Plan del Médico y la Enfermera de la Familia", con cobertura en todo el país; los Objetivos, Propósitos y Directrices en abril de 1992; el Movimiento de Comunidades Saludables en 1993; así como la Carpeta Metodológica en 1996, donde la promoción de salud ha estado presente en todas las acciones, según refiere Nélida Cruz. ${ }^{16,25,26}$

Dadas las referencias que en la Carta de Ottawa se hacen sobre el papel de la información y la comunicación en la promoción de la salud, se puede afirmar que en ella "se reconocía la decisiva importancia de la comunicación para ejecutar las políticas de promoción de la salud, estableciendo además que los medios de comunicación son actores claves para dicha promoción". 27

Luis Ramiro Beltrán citado por Nieto Olivar J M, define a la comunicación como el proceso de interacción social democrática, basado en el intercambio de símbolos, mediante el cual los seres humanos intercambian voluntariamente experiencias en condiciones de igualitario acceso, diálogo y participación. ${ }^{28}$

Considerando estos presupuestos teóricos, los resultados del análisis realizado permiten afirmar que el enfoque dado a los contenidos en estos trabajos apuntan a que en general, la salud es tratada desde la "medicalización", y el uso de tecnologías y no con un enfoque holístico positivo que integre al bienestar humano.

En cuanto a las áreas estratégicas de la promoción de la salud consideramos lo siguiente:

\section{Políticas públicas}

Tal vez la ausencia explícita de temas vinculados a políticas públicas en los medios se deba a su implementación como parte del proceso sociopolítico revolucionario cubano que data ya de más de 50 años, y que ha logrado consolidar un Sistema Nacional de Salud, universal, único y gratuito con altos presupuestos y prioridad en las políticas estatales. Por tanto, enunciar por ejemplo, la gratuidad de los servicios y su alcance equitativo, sería una reiteración informativa que ya "no es noticia". Esto no excluye la 
necesidad de explicar en el contexto actual estas políticas públicas, no ya en trabajos de información sino interpretativos, que aporten elementos en profundidad y ayuden al análisis de su significado sobre todo para el público más joven, además de abrir el diálogo en relación con las nuevas necesidades de salud, que demandan políticas públicas a tono con la realidad cubana actual, con independencia del nivel alcanzado por Cuba en materia de políticas de salud en el mapa mundial.

\section{Participación comunitaria}

En relación con la ausencia de alusión alguna a la participación de la comunidad en la salud, puede estar influido porque los artículos estudiados se refieren a acciones de salud que corresponden a los niveles terciarios y secundarios de atención, encargados de las investigaciones y la asistencia hospitalaria de alta especialización, pocos trabajos relativos a la atención primaria de salud más cercana a las comunidades en su entorno social y donde se dan mejor las condiciones para una mayor participación de los individuos. No obstante, la proyección de la participación en salud en los medios puede ser también reflejo del deterioro de una participación real y efectiva de la población en la toma de decisiones sobre salud, lo cual requeriría adentrarse en la dinámica de esta participación social y su proyección en los medios de comunicación, que trasciende al sector de la salud y a los propósitos de este estudio.

\section{Desarrollo de aptitudes personales}

Es el área en que más se encontraron trabajos publicados, que dan el mayor peso de la adopción de estilos de vida sanos y a la prevención de riesgos a los individuos.

\section{Creación de ambientes favorables}

En el tema sobre diversidad sexual es donde mayormente se aprecia esta área de la promoción de la salud, se demuestra la activación de mecanismos dialógicos, la creación de espacios de concertación y negociación y el estímulo a los derechos sociales de las personas, lo que contribuye también a la creación de un entorno social favorable para la promoción de la salud en este tema y a la vez denota una política pública en pos del derecho a la diversidad sexual.

\section{Reorientación de servicios de salud}

En este caso los trabajos se refieren a servicios de salud, no necesariamente a su reordenamiento, excluyendo la visión del "cambio de actitud y de organización de los servicios sanitarios, de forma que giren entorno a las necesidades del individuo como un todo, más allá de la mera responsabilidad de proporcionar servicios médicos", que afirman los expertos.

Se puede concluir que no se encontraron referencias a investigaciones con temas de salud en los medios digitales cubanos. La información sobre salud en los documentos seleccionados tuvo un marcado énfasis en promover la imagen de Cuba y el enfoque de divulgación no fue sustancial. No se demuestra una vinculación estrecha entre las áreas temáticas priorizadas por el MINSAP y los contenidos de los artículos publicados.

En cuanto a los tipos de contenidos publicados, en primer lugar, se situó la educación para la salud seguido de la asistencia sanitaria y servicios de salud, que en su mayoría se refirieron a servicios de atención terciaria y secundaria, y en tercer lugar, a las ciencias e investigaciones biomédicas y a fármacos, productos y tecnologías de la salud, vinculados a investigaciones. Fue destacada la mención de eventos 
científicos. Las alusiones a la economía de la salud, la formación en ciencias de la salud, las políticas públicas, fueron muy escasas, mientras que no se abordó a la cooperación internacional de salud desde la divulgación científica, todo lo cual fueron vacíos importantes, en el contexto cubano actual.

En general, se observó que la salud fue tratada desde la "medicalización" y el uso de tecnologías y no con un enfoque holístico positivo que integre al bienestar humano, lo que revela que el enfoque preventivo y, sobre todo, el de promoción de salud, tuvieron un considerable retraso en su proyección en los medios de comunicación estudiados, de acuerdo al más reciente paradigma de la salud, y en general, los trabajos estudiados no respaldaron las estrategias de la promoción de la salud.

Es recomendable realizar investigaciones sobre la representación de la salud en los medios digitales cubanos, desde la perspectiva de sus rutinas productivas e ideologías profesionales, y desde las necesidades y percepciones de los públicos, que permitan ampliar este estudio. Desarrollar productos y servicios de información especializada en salud, de calidad, en el entorno digital con independencia de los contenidos de información general; además de preservar y desarrollar los espacios dedicados a la salud en estos medios. Publicar contenidos de divulgación sobre salud en los medios digitales estudiados, con una mayor vinculación a las prioridades del MINSAP, sustentados en estrategias intersectoriales de información y comunicación social en ciencias de la salud. Diversificar los contenidos de divulgación especializada en los medios nacionales que comprendan a las políticas públicas, a la economía de la salud, la formación en ciencias de la salud y la cooperación internacional, entre otros, por su trascendencia en el contexto actual. Abogar por incorporar el enfoque de promoción de salud en los medios periodísticos digitales y publicar contenidos que respalden a sus estrategias.

\section{Anexo 1}

Dirección electrónica de las publicaciones revisadas

Granma: http://www.granma.cubaweb.cu

Juventud Rebelde: http://www.juventudrebelde.cu

Trabajadores: http://www.trabajadores.co.cu

Alma Mater: http://www.almamater.cu

Bohemia, edición mensual: http://www.bohemia.cubaweb.cu

Mujeres: $\underline{\text { http: //www. mujeres.co.cu }}$

Somos Jóvenes: http://www. somosjovenes.cu

\section{Anexo 2}

Lista de los documentos estudiados según medios. Abril 2009. 
Nota: se informa que al momento de la salida de este número, se comprobó que los documentos en los periódicos digitales Granma y Trabajadores no están accesibles en los URLs correspondientes, al parecer por problemas desconocidos por la autora.

\section{Granma}

Generalizarán empleo de biomaterial cubano en estomatología

http://www.granma.cubaweb.cu/2009/04/23/nacional/artic12.html

Expondrán programa cubano de trasplantes en cita mundial

http://www. granma.cubaweb.cu/2009/04/22/nacional/artic11.html

Creer en la esperanza

http://www.granma.cubaweb.cu/2009/04/25/nacional/artic01.html

Desarrollan algoritmos para el procesamiento digital de la señal electrocardiográfica

http://www.granma.cubaweb.cu/2009/04/23/nacional/artic16.html

Buenos resultados en la atención a la diabetes gestacional

http://www.granma.cubaweb.cu/2009/04/24/nacional/artic11.html

Exponen eficacia de vacunas cubanas contra el asma

http://www.granma.cubaweb.cu/2009/04/24/nacional/artic18.html

Refuerzan campesinos cubanos aportes a programa materno

http://www.granma.cubaweb.cu/2009/04/24/nacional/artic18.html

Médicos cubanos en Timor en jornada comunitaria 1 de mayo

http://www.granma.cubaweb.cu/2009/04/30/cubamundo/artic04.html

UNASUR constituye Consejo de Salud e impulsa Escudo Epidemiológico

http://www.granma.cubaweb.cu/2009/04/22/interna/artic05.html

Agradecen a Fidel Maestría en Asesoramiento Genético

http://www.granma.cubaweb.cu/2009/04/23/nacional/artic24.html

Alta mortalidad por malaria

http://www.granma.cubaweb.cu/2009/04/25/interna/01hilodirecto.html

Últimos avances de salud en cita mundial con sede en Cuba

http://www.granma.cubaweb.cu/2009/04/22/nacional/artic09.html

http://scielo.sld.cu 
Revista Cubana de Salud Pública. 2011;37(3):288-305

El CECMED no autoriza el uso humano del "veneno del escorpión azul" http://www. granma.cubaweb.cu/2009/04/24/nacional/artic03.html

Cubanos en maratón de asistencia médica en Guinea Bissau

http://www. granma.cubaweb.cu/2009/04/20/cubamundo/artic08.html

Saludables nacimientos en montañas del centro de Cuba

http://www.granma.cubaweb.cu/2009/04/21/nacional/artic08.html

Buscan poner límites a la influenza en México y países vecinos

http://www.granma.cubaweb.cu/2009/04/25/interna/artic10.html

\section{J uventud Rebelde}

¿Suave veneno?

http://www.juventudrebelde.cu/cuba/2009-04-19/suave-veneno/

Un arroz más nutritivo

http://www.juventudrebelde.cu/cuba/2009-04-19/un-arroz-mas-nutritivo/

Alientan ensayos clínicos contra tumores cerebrales

http://www.juventudrebelde.cu/cuba/2009-04-21/alientan-ensayos-clinicos-contratumores -cerebrales/

Exitosa prueba de prótesis en arterias cardíacas

http://www.juventudrebelde.cu/cuba/2009-04-23/exitosa-prueba-de-protesis-enarterias - cardiacas

Uno de cada dos habitantes del planeta padecerá alergia

http://www.juventudrebelde.cu/cuba/2009-04-24/uno-de-cada-dos-habitantes-delplaneta - padecera-alergia/

Garantizada la atención al paciente grave

http://www.juventudrebelde.cu/cuba/2009-04-24/garantizada-la-atencion-alpaciente-grave/

Abogan por extender cirugía de la epilepsia a niños

http://www.juventudrebelde.cu/cuba/2009-04-25/abogan-por-extender-cirugia-de-laepilepsia-a -ninos/

Adolescencia: ¿edad infeliz?

http://scielo.sld.cu 
Revista Cubana de Salud Pública. 2011;37(3):288-305

http://www.juventudrebelde.cu/cuba/2009-04-25/adolescencia-edad-infeliz/

Pregunte sin Pena I

http://www.juventudrebelde.cu/cuba/2009-04-25/adolescencia-edad-infeliz/

\section{Trabajadores}

Por la vida

http://www.trabajadores.cu/materiales_especiales/suplementos/salud/enfermedadescronicas-no-trasmisibles-1/a1por-la-vida

Santiago de Cuba: Sustituirán importación de hemoclasificadores y reactivos para el SIDA

http://www.trabajadores.cu/news/santiago-de-cuba-sustituiran-importacion-de hemoclasificadores-y-reactivos-para-el-sida

Psicoterapia bajo la lupa internacional en La Habana

http://www.trabajadores.cu/news/psicoterapia-bajo-la-lupa-internacional-en-la$\underline{\text { habana }}$

Biotecnología con economía

http://www.trabajadores.cu/materiales_especiales/columnistas/ramon-barrerasferran/biotecnologia-con-economia-para-columnistas/

Primer hospital de la Sierra Maestra

http://www.trabajadores.cu/news/primer-hospital-de-la-sierra-maestra

\section{Bohemia}

La mano mueve el mundo

http://www. bohemia.cubasi.cu/2009/04/24/especiales/manos.html

Extraño

http://www. bohemia.cubasi.cu/2009/04/22/unpoco/extrano.html

Técnicas médicas por la calidad de vida

http://www. bohemia.cubasi.cu/2009/04/18/nacionales/poder-popular-delegadosacontecer.html

Segunda etapa de vacunación antipolio

http://www. bohemia.cubasi.cu/2009/04/20/noticias/campana-vacunacionantipoliomelitis.html

http://scielo.sld.cu 
Revista Cubana de Salud Pública. 2011;37(3):288-305

\section{Mujeres}

El yogurt: Más beneficioso de lo que creemos

http: //www. mujeres. cubaweb.cu/articulo. asp?a $=2009 \&$ num $=434 \& a r t=60$

Herpes genital

http: //www. mujeres. cubaweb.cu/articulo.asp?a=2009\&num $=434 \& a r t=26$

Lechuga

http: //www. mujeres. cubaweb.cu/articulo.asp?a=2009\&num $=434 \& a r t=62$

En guardia contra la gordura

http://www. mujeres. cubaweb.cu/imprimir. asp?a=2009\&num $=434 \& a r t=28$

Hacer ejercicio exclusivamente no reduce riesgo de enfermedades cardíacas

http: //www. mujeres. cubaweb.cu/articulo.asp?a $=2009 \&$ num $=434 \& a r t=32$

Planificación familiar, métodos anticonceptivos y adolescencias

http: //www. mujeres. cubaweb.cu/articulo.asp?a $=2009 \&$ num $=433 \& a r t=30$

Tai Chi, Cambio de Vida

http: //www. mujeres. cubaweb.cu/articulo.asp?a $=2009 \&$ num $=434 \& a r t=30$

Consumo habitual de café puede disminuir riesgo de ictus

http: //www. mujeres. cubaweb.cu/articulo.asp?a $=2009 \&$ num $=434 \& a r t=34$

Día Mundial de lucha contra la hipertensión arterial: Actividades que se desarrollan en Cuba

http://www. mujeres. cubaweb.cu/actualidad.asp?art=3012

\section{Alma Mater}

Sabia la naturaleza, que nos hizo diferentes

http://www. almamater.cu/sitio\%20nuevo/paginas/voces/2009/abril/cenesex.html

\section{Somos J óvenes}

Celebró Cuba Día Mundial de Lucha contra la Tuberculosis

http://www. somosjovenes.cu/index/semana138/tuberculos.htm

Nota: Los siguientes trabajos de Mujeres, no se incluyen por ser rebotes de sus homólogos en otros medios.

http://scielo.sld.cu 
Revista Cubana de Salud Pública. 2011;37(3):288-305

Saludables nacimientos en montañas de Cuba

http://www. mujeres.cubaweb.cu/actualidad.asp?art=3016

Segunda etapa antipolio

http://www. mujeres. cubaweb.cu/actualidad.asp?art=3014

\section{REFERENCI AS BI BLI OGRÁFICAS}

1. Catálogo Nacional de Publicaciones Seriadas [Internet]. La Habana: Catálogo [citado 28 Feb 2009]. Disponible en: http://www.seriadas.cult.cu

2. Pereira JM. Comunicación, desarrollo y promoción de la salud: Enfoques, balances y desafíos [Internet]. [citado 16 Abr 2009]. Disponible en:

http://www.comminit.com/es/node/149881

3. Calvo Hernando M. Repensar las estrategias de comunicación en la promoción de la salud [Internet]. Sevilla: Universidad de Sevilla, España [citado 19 de May 2009].

Disponible en

http://74.125.95.132/search?q=cache: NWxKNXiWI lo] : www. hapaxmedia. net/ibercom /pdf/CalvoCalvoManuelAngel. pdf+comunicacion+OR+medios+

OR+masivos+OR+prensa+OR+divulgacion $+\% 22$ promocion + de + salud $\% 22 \& c d=46 \& h 1$ $=\mathrm{es} \& \mathrm{ct}=\mathrm{clnk} \& \mathrm{gl}=\mathrm{cu}$

4. López Beltrán C. La comunicación de la ciencia revisitada. En: Estrada Martínez L, editor. La divulgación de la ciencia: ¿ecuación, apostolado o...? México, D.F.:

Dirección General de Divulgación de la Ciencia, UNAM; 2003. p.18-25.

5. Moreno Aragón J. Aproximación teórica a las bases epistemológicas de la Comunicación de la Ciencia y la Tecnología. En: Trilles I, Rodríguez M, editores coordinadores. La Comunicación de la Ciencia y la Tecnología: una visión universitaria. La Habana: Dirección de Extensión Universitaria, MES; 2005. p.60.

6. Marin Murillo F, Armentia Vizuete JI. El Periodismo científico en los diarios digitales del Estado Español [Internet]. Bilbao, España: Universidad del País Vasco; 2002 [citado 19 May 2009]. Disponible en:

http://redalyc.uaemex.mx/pdf/819/81996107.pdf

7. Moreno Castro C. Comunicación y divulgación de la ciencia. Comunicar. 2002 [citado 19 de Abr 2002]; (9). Disponible en:

http://www.revistacomunicar.com/index.php?contenido=detalles\&numero=19\&articul $\underline{0=19-2002-04}$

8. Orihuela J L. Los 10 paradigmas de la e-Comunicación [Internet]. [citado 29 May 2009]. Disponible en:

http://www.agetec.org/ageteca/Los $\% 2010$ Paradigmas $\% 20$ de $\% 201 a \% 20$ e-

Comunicacion.pdf

9. Aportes para la Formulación de una Política de Comunicación para la Promoción de la Salud en América [Internet]. [citado 28 Mar 2009]. Disponible en:

http://www.comminit.com/es/node/150392

http://scielo.sld.cu 


\section{Revista Cubana de Salud Pública. 2011;37(3):288-305}

10. González De Haro MD. La salud y sus implicaciones sociales, políticas y Educativas. Huelva: Servicio de Publicaciones de la Universidad de Huelva; 2006.

11. Proyecciones de la Salud Pública en Cuba para el 2015. La Habana: MINSAP; 2006.

12. Documentos generales de Promoción de la Salud [Internet]. [citado 15 Mar 2009]. Disponible en: http://www. medicinafamiliaruc.cl/html / promocion salud.html

13. Álvarez Sintes R. Temas de Medicina General Integral. La Habana: Editorial Ciencias Médicas; 2001.

14. Laplacette. Proceso Salud/enfermedad/atención [Internet]. 2008 [citado 8 Abr 2009]. Disponible en:

http://www. unanleon. edu. ni/facultades/medicina/pregrado/descarga/servicio_social/ mgi_volii.pdf

15. Promoción de salud [Internet]. [citado 11 Ene 2009]. Disponible en:

http://www. monografias.com/trabajos64/promocion-salud/promocion - salud2.shtml

16. Cruz Álvarez NM, Vázquez Vigoa A. Algunas consideraciones sobre promoción de salud. RESUMED [Internet]. 2001[citado 29 May 2009]; 14(5):205-10. Disponible en: http://bvs.sld.cu/revistas/res/vol14_5_01/res01501.htm

17. Arango Panesso Y. Referentes socio-históricos latinoamericanos: contribución a los fundamentos políticos de la promoción de salud. Rev Cubana Salud Pública [Internet]. 2008 [citado 29 May 2009]; 34 (1). Disponible en:

http://scielo.sld.cu/scielo.php?script=sci_arttext\&pid=S0864 $\underline{34662008000100017 \& \operatorname{lng}=\mathrm{es}}$

18. Toledo Curbelo GJ. Sistemas de salud y estrategias para solucionar los problemas de salud. La Habana: Editorial Ciencias Médicas; 1999.

19. Promoción de la salud: una antología. Washington, D.C.: OPS; 1999

20. Restrepo H. Promoción de la salud: Cómo construir vida saludable. Bogotá: Editorial Médica Panamericana; 2001.

21. . Las políticas de promoción de la salud en la Organización Panamericana de la Salud. Conferencia Internacional de Promoción de la Salud. Colombia, Santa Fe de Bogotá: OPS; 1992.

22. Promoción de la salud: una antología. OPS. Washington, D.C.: 1996.

23. OPS/OMS. Carta de Otawa para la promoción de salud. Conferencia Internacional sobre Promoción de Salud, Otawa. 17-21 de noviembre de 1986. Washington, D.C.: OPS/OMS; 1987.

24. Carta de Ottawa [Internet]. Washington, D.C.: OPS/OMS [citado 18 Abr 2009]. Disponible en: http://www. buenastareas.com/ensayos/Carta-De-Otawa/84543. html

25. Piñón Vega J, Torres Hernández M. Impacto de la promoción y la educación para la salud en los logros de la salud pública cubana. Rev Cubana Med Gen Integr [Internet]. 2001 [citado 9 May 2009]; 17 (1): 91-9. Disponible en: http://bvs.sld.cu/revistas/mgi/vol17_1_01/mgi13101.htm

http://scielo.sld.cu 
26. Políticas, Estrategias y Programas Priorizados del Sistema Nacional de Salud [Internet]. La Habana: Dirección Nacional de Estadísticas, MINSAP; 1998 [citado 4 Feb 2009]. Disponible en: http://www.sld.cu/sistema_de_salud/estrategias.html

27. Coe G. Comunicación y promoción de la salud. Revista Latinoamericana Comunicación [Internet]. 1998 [citado 21 May 2009];(63). Disponible en: http://www.comunica.org/chasqui/coe.htm

28. Nieto Olivar J M. Para intentar encontrarnos: comunicación educativa en la promoción de derechos humanos sexuales y reproductivos con adolescentes. UNI Revista [Internet]. [citado 21 May 2009]; 1(3). Disponible en: http://www. alaic.net/ponencias/UNIrev_Nieto.pdf

Recibido: 6 de abril de 2010.

Aprobado: 18 de enero de 2011.

Alina Pérez Martínez. Centro Nacional de Información de Ciencias Médicas. Calle 27 No. 110 e M y N. El Vedado 10400. La Habana, Cuba. Teléf.: 8330274. Correo electrónico: aliperez@infomed.sld.cu 